\title{
Role of the neural niche in brain metastatic cancer
}

John Termini, $\mathbf{P h D}^{1}$, Josh Neman, $\mathbf{P h D}^{2}$, and Rahul Jandial, $\mathbf{M D}, \mathbf{P h D}^{2,3}$

${ }^{1}$ Department of Molecular Medicine, City of Hope, Duarte, CA 91010

2Division of Neurosurgery, City of Hope, Duarte, CA 91010

${ }^{3}$ Department of Biology, University of Southern California, Los Angeles, CA 90089

\section{Abstract}

Metastasis is the relenteless pursuit of cancer to escape its primary site and colonize distant organs. This malignant evolutionary process is biologically heterogeneous, yet one unifying element is the critical role of the microenvironment for arriving metastatic cells. Historically brain metastases were rarely investigated since patients with advanced cancer were considered terminal. Fortunately, advances in molecular therapies have led to patients living longer with metastatic cancer. However, one site remains recalcitrant to our treatment efforts - the brain. The central nervous system is the most complex biological system, which poses unique obstacles but also harbors opportunities for discovery. Much of what we know about the brain microenvironment comes from neuroscience. We suggest that the interrelated cellular responses in traumatic brain injury may guide us towards new perspectives in understanding brain metastases. In this view, brain metastases may be conceptualized as progressive oncologic injury to the nervous system. This review discusses our evolving understanding of the bidirectional interactions between the brain milieu and metastatic cancer.

\section{Keywords}

brain metastasis; tumor microenvironment; neural niche

\section{INTRODUCTION}

Metastases are responsible for the majority of deaths from cancer. Dissemination of primary cancers to secondary organ sites is most ominous when tumors spread to the brain. Brain metastases indicate poor prognosis, likely exclusion from clinical trials, and cause neurological deficits that impair patient's lives in fundamental ways. Existing therapies are restricted to palliative radiation and neurological surgery, with limited chemotherapeutic options (1).

As the global incidence of cancer has increased, brain metastases now appear in 8-10\% of all cases $(2,3)$. Several factors underlie this epidemiological shift. Major advances in neuroimaging have led to increased detection of brain metastases in patients (4).

Corresponding Author: Rahul Jandial, MD, PhD, Division of Neurosurgery, City of Hope, 1500 East Duarte Rd, Duarte, CA, 91010 , Telephone: 1-626-256-4673, rjandial@ coh.org. 
Concomitantly, while targeted therapies have improved management of systemic disease, poor bioavailability to the brain increases its potential as a sanctuary site for metastatic disease. Fortunately cancer patients are living longer, but extended survival from primary disease has unmasked brain metastases as one of the major challenges limiting outcomes for those with advanced disease (5).

Metastasis is not the biological narrative of an autonomous cell. More likely it is a dynamic interplay between neoplastic cells and newly encountered microenvironments. Traditionally metastatic spread was conceived as a late event occurring long after primary tumors progressed locally (6). This may still be true for some tumors; however, recent evidence also suggests parallel progression of primary and metastatic disease. Indeed, circulating tumor cells (CTCs) can be detected in newly diagnosed early stage cancer (6). In spite of this patients may progress to clinically detectable brain metastases years to decades after primary diagnosis.

As CTCs course through the cerebral vasculature, the blood brain barrier (BBB) poses an innate obstacle to extravasation (7). Although formidable, the BBB is penetrable. CTCs can effectively (although inefficiently) cross the intact BBB and form micrometastases (8). The ability of cells to traverse the BBB, as well as the clinical latency between the appearance of early circulating cells and development of brain metastases raises an intriguing biological question. Could the rate-limiting event in the formation of brain metastases be the last step of the metastatic cascade -- colonization of the neural niche?

After crossing the BBB, disseminated primary tumor cells arrive in a dynamic cellular and molecular landscape that presents unique selection pressures. There is a paucity of research on brain metastases from this perspective, which offers an opportunity for new discoveries to extend the quality and duration of patient's lives. This review will focus on the reciprocal interactions between brain metastases and the neural niche.

\section{DEFINING THE BRAIN MILIEU}

Unique cell types comprise the brain milieu. Neurons are the principal components of the central nervous system (CNS), and are electrically excitable cells that use neurotransmitters in synaptic connections for communication. Five classical neurotransmitters (glutamate, GABA, serotonin, acetylcholine, and dopamine) are released from pre-synaptic vesicles and activate respective receptors on post-synaptic neurons (9). Viability of neurons is maintained by endogenous growth factors called neurotrophins (NGF, BDNF, NT-3, NT-4), which regulate cell fate, growth, and plasticity (10). Neurotrophins also modulate germinal zones that are repositories of neural stem cell (NSC) niches in the adult brain (the subgranular zone of the dentate gyrus in the temporal lobes and the subventricular zone of the frontal lobes) $(11,12)$. As neurons acquire the ability to generate action potentials and communicate by synaptic transmission they lose many essential metabolic pathways (13). As a consequence, the adult brain relies on microenvironmental metabolic support from surrounding glial cells.

Homeostasis in the brain microenvironment is maintained by supportive glial cells called astrocytes (14). Branching astrocytic processes cloak most cellular components throughout the CNS, including the BBB. In the uninjured CNS, astrocytes play an essential role in the 
maintenance of extracellular ionic environment and $\mathrm{pH}$, clearance and release of extracellular glutamate, and provision of metabolic substrates for neurons (15). Purinergic signaling is the most important pathway by which astrocytes communicate with other cells (16). This includes ATP as the main transmitter for communication among neighboring astrocytes. ATP is also important in paracrine signaling to neurons, blood vessels, and microglial cells. The other prominent glial cells in the CNS are the myelin producing oligodendrocytes. A single oligodendrocyte can extend its processes to 50 axons, wrapping each with approximately $1 \mu \mathrm{m}$ of myelin sheath. In addition, oligodendrocytes can receive synaptic inputs from neurons an participate in metabolic homeostasis of the brain milieu (17, 18).

\section{INSIGHTS FROM NEUROSCIENCE}

Our understanding of how the brain responds to injury has grown dramatically in recent years. This will likely yield new ideas about the relationship between metastatic cells and the neural niche. Traumatic brain injury (TBI) disrupts normal circuitry by causing neuronal and glial cell death, inducing a cascade of microenvironmental perturbations (19). TBI damages the biosynthetic capacity of neurons to make GABA, and can lead to a toxic accumulation of the glutamate precursor (20). This disruption in the balance of GABA and glutamate is associated with increased epileptic brain activity increasing the potential for additional damage.

The brain can limit the spread of injury by forming a glial scar to seal off damaged areas. In the adult brain, astrocytes are typically quiescent and not proliferative unless activated by disease (21). These "reactive" astrocytes respond with hypertrophy and increased expression of intermediate filament glial fibrillary acidic protein (GFAP) in a process termed gliosis. Experimental ablation of proliferating reactive astrocytes disrupts scar formation causing increased neuronal loss and demyelination (15). This exacerbates clinical deficits and impairs functional recovery, supporting the essential role of glial scar forming astrocytes in neural protection and repair. The astrocytic response to injury is supplemented by resident oligodendrocyte progenitor cells, which participate in gliosis and regeneration. Oligodendrocyte progenitor cells locally secrete chondroitin sulphate proteoglycan (NG2) which diminishes axonal re-growth (22).

\section{NEOPLASTIC ADAPTATIONS TO THE NEURAL NICHE}

Cancer cells arrive in the neural niche to execute the last step in the metastatic cascade colonization (23). It is unknown whether cells already possess latent traits that facilitate brain colonization or if advantageous mutations are acquired under neural niche selection pressures $(24,25)$. It may be possible that primary tumors evolve and release cells with brain-trophic mutations at an appreciable frequency to succeed despite the inefficient process of metastasis. Alternatively, pre-metastatic cells may evolve under new microenvironmental pressures after their arrival in the brain that drives fate determination.

It is more plausible that some combination of these strategies takes place. Tumor cells may survive by exploiting a period of dormancy allowing for the development of bidirectional interactions that transform the local brain milieu into a hospitable niche. Once this favorable 
environment is achieved they could escape dormancy and progress to clinically relevant macrometastases. Given the heterogeneous patterns observed in the clinical progression of brain metastases, tumors most likely employ varied approaches that are specific to a patient's biology and history of medical intervention. In all scenarios the brain's microenvironment provides the critical selection pressure.

The role of astrocytes in brain metastases has been defined by seminal contributions from several groups $(8,26-33)$. Early work demonstrated the role of paracrine signaling via cytokine release by astrocytes in the facilitation of brain metastases. In addition to releasing various interleukins, reactive astrocytes up regulate survival genes (GSTA5, BCL2L1, TWIST1) in breast cancer cells resulting in resistance to chemotherapeutic agents (34). Furthermore, with direct relevance to mechanisms of treatment efficacy, inhibition of PDGFR $\beta+$ astrocyte sub groups with Pazopanib prevented the outgrowth of Her2+ brain metastases in animal models (35). The indispensable contribution of astrocytes to metastatic colonization is well established and continues to be robustly investigated.

The ability of metastatic cells to alter the physiologic microenvironment of the brain towards a tumor-favorable microenvironment was recently described by our group (36). Since NSCs can differentiate into neurons or glial cells based on temporal and molecular signals arising during development or brain injury, their role in metastasis was investigated. Histological interrogation of neurosurgical specimens identified NSCs in breast metastases and adjacent brain. Reactive astrocytes were also seen, and interestingly they resembled those found in glial scars of patients with TBI. Moreover, metastatic cells overexpressed BMP-2, which is also known to be a strong molecular cue for NSC differentiation into astrocytes. Co-culture experiments with metastatic cells from the surgical specimens and NSCs showed tumor cell growth is inhibited by NSCs initially. However, over time metastatic cells were able to escape NSC inhibition by steering them to an astrocytic lineage through paracrine BMP-2 signaling.

The effect of neurotransmitters on brain metastases has also been explored (37). Comparison of primary and brain metastatic tissue and cells revealed upregulation of both cell surface GABA transporters and receptors. Although it was anticipated that GABA would potentiate growth through receptor signaling, experiments revealed an energetic role as a metabolic substrate following cytosolic shuttling through GABA transporters. A proliferative advantage was conferred by the ability of metastatic cells to take up and catabolize GABA with the resultant formation of NADH through the GABA-shunt. Together these findings suggest that cancers may survive in the brain microenvironment by co-opting neurotransmitters as oncometabolites.

The brain's extracellular space is rife with growth factors which maintain cellular homeostasis (38). These growth factors could serve as powerful substrates for metastatic tumor cells that possess or evolve the ability to utilize the associated signaling pathways (39, 40). This would be particularly advantageous in the context of metastatic dormancy or micrometastasis where angiogenesis is sparse. Our preliminary findings suggest that tumor cells express neurotrophin receptor TrkB and are selectively activated by BDNF from the brain microenvironment. Recognizing that breast cancer metastasizes to the brain in 
approximately $40 \%$ of Her2+ patients we are investigating a possible interaction between TrkB and Her2+ (41). Our preliminary results show BDNF induces TrkB-Her2 dimerization with co-localization of Her2 and phosphorylated TrkB receptor in newly derived metastatic cells (unpublished). The cooperation of neurotrophin and Her2 receptors could result in amplified oncogenic signaling from transactivation of both receptors with BDNF. Given the abundance of neurotrophins in the neural niche, this could partly explain the proclivity of breast cancer to spread to the brain in Her2+ patients.

The brain milieu most likely selects for metastatic cells that are best able to overcome natural barriers to infiltration, as well asutilize the nutrient resources and signaling pathways of the brain. Metastatic cells that cross the BBB and overexpress BMP-2 could modify the local cellular milieu by differentiating NSCs into astrocytes. The innate and amplified astrocytic response provides cytokine stimulation to tumor cells that could allow early survival as dormant or micrometastases. With time, some cells may acquire a more neural phenotype with expression of classical neuronal receptors and signaling pathways allowing further growth advantage. Ultimately the progressive selection and evolution of metastatic cells could allow for extensive colonization of the brain and subsequent neurological injury.

\section{CENTRAL NERVOUS eco-SYSTEM}

For nearly a century the "no new neuron" theory was held as dogma, suggesting the brain is unable to regenerate with little plasticity remaining after developmental maturity $(42,43)$. With the discovery of germinal niches in the brain, the notion of the brain as immutable has been shed and a renaissance in neuroscience has occurred. New methods such as "optogenetics" are allowing neuroscientists to control the activity of neuronal groups with extraordinary precision $(44,45)$. New insights about the anatomical and functional architecture of the human prenatal brain are revealing transcriptional landscapes that may eventually guide neural repair in the context of oncologic injury (46).

In parallel the importance of the tumor microenvironment has been established in cancer biology. Despite originating in disparate germ line lineages, tumor cells are able to inhabit the neural niche. This ability to adapt to and possibly co-opt the brain's natural resources demonstrates the resilience and adaptability of cancer. Borrowing from ecological principles, metastasis could be an adaptive radiation of neoplastic cells from the primary tumor $(47,48)$. Each malignant scout having the opportunity to undergo its own divergent evolution and create distinct metastatic species (49).

The proverbial "soil" of the brain is a unique and mostly unchartered environment. It is likely that technological and creative advancements in neuroscience will reveal unanticipated biological complexities and clinical opportunities in metastatic brain tumors.

\section{Acknowledgments}

Financial Support. John Termini is supported by NIH grant R01CA176611-01. Josh Neman is supported by California Institute for Regenerative Medicine (CIRM) grant TG2-01150. Rahul Jandial is supported by National Institutes of Health (NIH) grant 2K12CA001727-16A1 


\section{References}

1. Hambrecht A, Jandial R, Neman J. Emerging role of brain metastases in the prognosis of breast cancer patients. Breast Cancer (Dove Med Press). 2011; 3:79-91. [PubMed: 24367178]

2. Neman J, Somlo G, Jandial R. Classification of genomic changes in breast cancer brain metastasis. Neurosurgery. 2010; 67:N18-9. [PubMed: 20644404]

3. Barnholtz-Sloan JS, Sloan AE, Davis FG, Vigneau FD, Lai P, Sawaya RE. Incidence proportions of brain metastases in patients diagnosed (1973 to 2001) in the Metropolitan Detroit Cancer Surveillance System. J Clin Oncol. 2004; 22:2865-72. [PubMed: 15254054]

4. Lin NU, Amiri-Kordestani L, Palmieri D, Liewehr DJ, Steeg PS. CNS metastases in breast cancer: old challenge, new frontiers. Clin Cancer Res. 2013; 19:6404-18. [PubMed: 24298071]

5. Owonikoko TK, Arbiser J, Zelnak A, Shu HK, Shim H, Robin AM, et al. Current approaches to the treatment of metastatic brain tumours. Nat Rev Clin Oncol. 2014; 11:203-22. [PubMed: 24569448]

6. Comen E, Norton L, Massague J. Clinical implications of cancer self-seeding. Nat Rev Clin Oncol. 2011; 8:369-77. [PubMed: 21522121]

7. Connell JJ, Chatain G, Cornelissen B, Vallis KA, Hamilton A, Seymour L, et al. Selective permeabilization of the blood-brain barrier at sites of metastasis. J Natl Cancer Inst. 2013; 105:1634-43. [PubMed: 24108809]

8. Lockman PR, Mittapalli RK, Taskar KS, Rudraraju V, Gril B, Bohn KA, et al. Heterogeneous blood-tumor barrier permeability determines drug efficacy in experimental brain metastases of breast cancer. Clin Cancer Res. 2010; 16:5664-78. [PubMed: 20829328]

9. Lauder JM. Neurotransmitters as growth regulatory signals: role of receptors and second messengers. Trends Neurosci. 1993; 16:233-40. [PubMed: 7688165]

10. Lu B, Pang PT, Woo NH. The yin and yang of neurotrophin action. Nat Rev Neurosci. 2005; 6:603-14. [PubMed: 16062169]

11. Alvarez-Buylla A, Garcia-Verdugo JM. Neurogenesis in adult subventricular zone. J Neurosci. 2002; 22:629-34. [PubMed: 11826091]

12. Temple S. The development of neural stem cells. Nature. 2001; 414:112-7. [PubMed: 11689956]

13. Herrero-Mendez A, Almeida A, Fernandez E, Maestre C, Moncada S, Bolanos JP. The bioenergetic and antioxidant status of neurons is controlled by continuous degradation of a key glycolytic enzyme by APC/C-Cdh1. Nat Cell Biol. 2009; 11:747-52. [PubMed: 19448625]

14. Ota Y, Zanetti AT, Hallock RM. The role of astrocytes in the regulation of synaptic plasticity and memory formation. Neural Plast. 2013; 2013:185463. [PubMed: 24369508]

15. Sofroniew MV, Vinters HV. Astrocytes: biology and pathology. Acta Neuropathol. 2010; 119:735. [PubMed: 20012068]

16. Pascual O, Casper KB, Kubera C, Zhang J, Revilla-Sanchez R, Sul JY, et al. Astrocytic purinergic signaling coordinates synaptic networks. Science. 2005; 310:113-6. [PubMed: 16210541]

17. Morrison BM, Lee Y, Rothstein JD. Oligodendroglia: metabolic supporters of axons. Trends Cell Biol. 2013; 23:644-51. [PubMed: 23988427]

18. Neman J, de Vellis J. A method for deriving homogenous population of oligodendrocytes from mouse embryonic stem cells. Dev Neurobiol. 2012; 72:777-88. [PubMed: 22234971]

19. Sharp DJ, Scott G, Leech R. Network dysfunction after traumatic brain injury. Nat Rev Neurol. 2014; 10:156-66. [PubMed: 24514870]

20. Ylikoski J, Mrena I, Panula P, Happola O, Paivarinta H. Neural control of the middle ear mucosa. Acta Otolaryngol Suppl. 1989; 457:159-63. [PubMed: 2467506]

21. Anderson MA, Ao Y, Sofroniew MV. Heterogeneity of reactive astrocytes. Neurosci Lett. 2014; 565C:23-9. [PubMed: 24361547]

22. Busch SA, Horn KP, Cuascut FX, Hawthorne AL, Bai L, Miller RH, et al. Adult NG2+ cells are permissive to neurite outgrowth and stabilize sensory axons during macrophage-induced axonal dieback after spinal cord injury. J Neurosci. 2010; 30:255-65. [PubMed: 20053907]

23. Langley RR, Fidler IJ. The biology of brain metastasis. Clin Chem. 2013; 59:180-9. [PubMed: 23115057] 
24. Fidler IJ, Kripke ML. Metastasis results from preexisting variant cells within a malignant tumor. Science. 1977; 197:893-5. [PubMed: 887927]

25. Talmadge JE, Wolman SR, Fidler IJ. Evidence for the clonal origin of spontaneous metastases. Science. 1982; 217:361-3. [PubMed: 6953592]

26. Palmieri D, Smith QR, Lockman PR, Bronder J, Gril B, Chambers AF, et al. Brain metastases of breast cancer. Breast Dis. 2006; 26:139-47. [PubMed: 17473372]

27. Palmieri D, Chambers AF, Felding-Habermann B, Huang S, Steeg PS. The biology of metastasis to a sanctuary site. Clin Cancer Res. 2007; 13:1656-62. [PubMed: 17363518]

28. Palmieri D, Bronder JL, Herring JM, Yoneda T, Weil RJ, Stark AM, et al. Her-2 overexpression increases the metastatic outgrowth of breast cancer cells in the brain. Cancer Res. 2007; 67:41908. [PubMed: 17483330]

29. Fitzgerald DP, Palmieri D, Hua E, Hargrave E, Herring JM, Qian Y, et al. Reactive glia are recruited by highly proliferative brain metastases of breast cancer and promote tumor cell colonization. Clin Exp Metastasis. 2008; 25:799-810. [PubMed: 18649117]

30. Fidler IJ, Balasubramanian K, Lin Q, Kim SW, Kim SJ. The brain microenvironment and cancer metastasis. Mol Cells. 2010; 30:93-8. [PubMed: 20799011]

31. Bos PD, Zhang XH, Nadal C, Shu W, Gomis RR, Nguyen DX, et al. Genes that mediate breast cancer metastasis to the brain. Nature. 2009; 459:1005-9. [PubMed: 19421193]

32. Kim MY, Oskarsson T, Acharyya S, Nguyen DX, Zhang XH, Norton L, et al. Tumor self-seeding by circulating cancer cells. Cell. 2009; 139:1315-26. [PubMed: 20064377]

33. Valiente M, Obenauf AC, Jin X, Chen Q, Zhang XH, Lee DJ, et al. Serpins promote cancer cell survival and vascular co-option in brain metastasis. Cell. 2014; 156:1002-16. [PubMed: 24581498]

34. Kim SJ, Kim JS, Park ES, Lee JS, Lin Q, Langley RR, et al. Astrocytes upregulate survival genes in tumor cells and induce protection from chemotherapy. Neoplasia. 2011; 13:286-98. [PubMed: 21390191]

35. Gril B, Palmieri D, Qian Y, Anwar T, Liewehr DJ, Steinberg SM, et al. Pazopanib inhibits the activation of PDGFRbeta-expressing astrocytes in the brain metastatic microenvironment of breast cancer cells. Am J Pathol. 2013; 182:2368-79. [PubMed: 23583652]

36. Neman J, Choy C, Kowolik CM, Anderson A, Duenas VJ, Waliany S, et al. Co-evolution of breast-to-brain metastasis and neural progenitor cells. Clin Exp Metastasis. 2013; 30:753-68. [PubMed: 23456474]

37. Neman J, Termini J, Wilczynski S, Vaidehi N, Choy C, Kowolik CM, et al. Human breast cancer metastases to the brain display GABAergic properties in the neural niche. Proc Natl Acad Sci U S A. 2014; 111:984-9. [PubMed: 24395782]

38. Lu B, Nagappan G, Guan X, Nathan PJ, Wren P. BDNF-based synaptic repair as a diseasemodifying strategy for neurodegenerative diseases. Nat Rev Neurosci. 2013; 14:401-16. [PubMed: 23674053]

39. Ricci A, Greco S, Mariotta S, Felici L, Bronzetti E, Cavazzana A, et al. Neurotrophins and neurotrophin receptors in human lung cancer. Am J Respir Cell Mol Biol. 2001; 25:439-46. [PubMed: 11694449]

40. Louie E, Chen XF, Coomes A, Ji K, Tsirka S, Chen EI. Neurotrophin-3 modulates breast cancer cells and the microenvironment to promote the growth of breast cancer brain metastasis. Oncogene. 2013; 32:4064-77. [PubMed: 23001042]

41. Wolff AC, Hammond ME, Hicks DG, Dowsett M, McShane LM, Allison KH, et al. Recommendations for human epidermal growth factor receptor 2 testing in breast cancer: American Society of Clinical Oncology/College of American Pathologists clinical practice guideline update. J Clin Oncol. 2013; 31:3997-4013. [PubMed: 24101045]

42. Sotelo C. Viewing the brain through the master hand of Ramon y Cajal. Nat Rev Neurosci. 2003; 4:71-7. [PubMed: 12511863]

43. Ramón y Cajal S. Sobre las fibras nerviosas de la capa molecular del cerebelo. Rev Trim Histol Normal Patol. 1888; 1:33-49.

44. Bass CE, Grinevich VP, Gioia D, Day-Brown JD, Bonin KD, Stuber GD, et al. Optogenetic stimulation of VTA dopamine neurons reveals that tonic but not phasic patterns of dopamine 
transmission reduce ethanol self-administration. Front Behav Neurosci. 2013; 7:173. [PubMed: 24324415]

45. Nelson AB, Hammack N, Yang CF, Shah NM, Seal RP, Kreitzer AC. Striatal Cholinergic Interneurons Drive GABA Release from Dopamine Terminals. Neuron. 2014; 82:63-70. [PubMed: 24613418]

46. Miller JA, Ding SL, Sunkin SM, Smith KA, Ng L, Szafer A, et al. Transcriptional landscape of the prenatal human brain. Nature. 2014; 508:199-206. [PubMed: 24695229]

47. Martin $\mathrm{CH}$, Wainwright PC. Multiple fitness peaks on the adaptive landscape drive adaptive radiation in the wild. Science. 2013; 339:208-11. [PubMed: 23307743]

48. Flaxman SM, Wacholder AC, Feder JL, Nosil P. Theoretical models of the influence of genomic architecture on the dynamics of speciation. Mol Ecol. 2014

49. Ostrow SL, Barshir R, DeGregori J, Yeger-Lotem E, Hershberg R. Cancer evolution is associated with pervasive positive selection on globally expressed genes. PLoS Genet. 2014; 10:e1004239. [PubMed: 24603726]

50. Sierra A, Price JE, Garcia-Ramirez M, Mendez O, Lopez L, Fabra A. Astrocyte-derived cytokines contribute to the metastatic brain specificity of breast cancer cells. Lab Invest. 1997; 77:357-68. [PubMed: 9354770] 


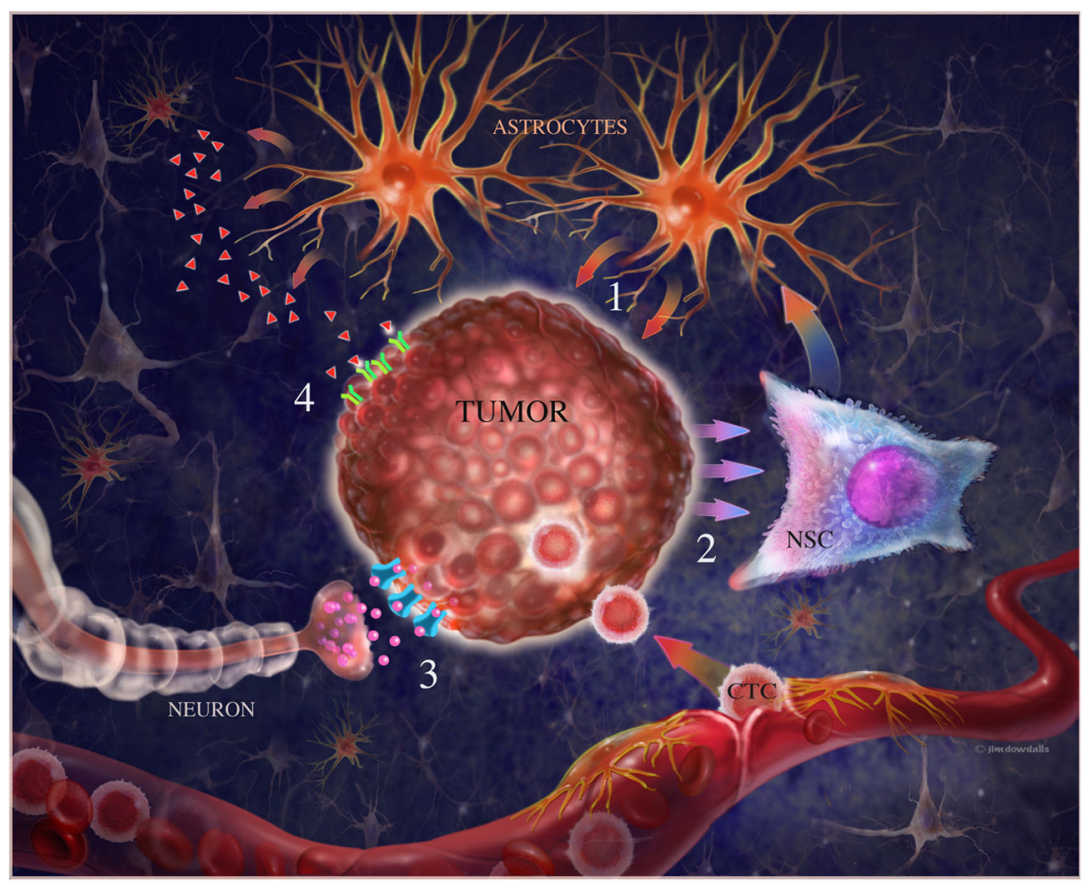

Figure 1. Interactions between metastatic cells and the brain milieu

1) Astrocytes release cytokines that facilitate colonization by stimulating growth of tumor cells (50). 2) Brain metastates release BMP-2 which increase astrocytic density by differentiating NSCs toward astrocytic lineage (36). 3) Neurons release GABA neurotransmitters that shuttle through their respective transporters to serve as metabolic substrates (37). 4) Possibly, abundant neurotrophins in the brain's extracellular space are captured by their cognate receptors on metastatic cells (unpublished). CTC $=$ circulating tumor cells, $\mathrm{NSC}=$ neural stem cells. 